\title{
PROPERTIES OF NORMAL AND SUPERCONDUCTING STATES OF DIFFERENTLY DOPED REBa $\mathrm{Cu}_{3} \mathrm{O}_{7-x}$
}

\author{
C. SulKowski \\ Institute of Low Temperature and Structure Research, Polish Academy of Sciences \\ P.O. Box 937, 50-950 Wrocław, Poland
}

(Received January 5, 1995; revised version May 25, 1995)

\begin{abstract}
Temperature dependence of resitivity, $\rho(T)$, thermopower, $S_{300}$, superconducting transition temperature, $T_{c}$, and lower critical field, $H_{\mathrm{c} 1}(0)$, of differently doped $\mathrm{REBa}_{2} \mathrm{Cu}_{3} \mathrm{O}_{7-x}$ ceramics have been measured. The hole doping of the $\mathrm{CuO}_{2}$ plane is determined by the $T_{c}$, the oxygen concentration and/or the value of the thermopower. The temperature dependence of the resistivity above $200 \mathrm{~K}$ and the values of the lower critical field $H_{\mathrm{c} 1}(0)$ show clear dependence on the hole concentration. Below $200 \mathrm{~K}$, in contrast to simple metals, the resitivity of all samples decreases faster than linearly with the decreasing temperature. Classical electron-phonon scattering mechanism is not appropriate to describe the $\rho(T)$ curves of $\mathrm{REBa}_{2} \mathrm{Cu}_{3} \mathrm{O}_{7-x}$ compounds. Some observed features of the $\rho(T)$ dependence are predicted by mean field phase diagram for the $\mathrm{CuO}_{2}$ plane.
\end{abstract}

PACS numbers: 74.72.-h, 74.25.Fy

\section{Introduction}

Transport properties in the normal state of high- $T_{\mathrm{c}}$ superconductors are inconsistent with a description by the conventional electron-phonon scattering mechanism. These properties are one of the most attractive subjects for investigation, since the excitation which interacts with carriers might play an important role in superconductivity [1]. Especially the normal state resistivity reveals an anomalous feature. Hole concentration in $\mathrm{CuO}_{2}$ plane depends mainly on doping from carriers reservoir, i.e., the $\mathrm{CuO}$ chains [2]. The doping of $\mathrm{CuO}_{2}$ plane increases with oxygen concentration. Opposite change in carriers concentration could be caused by substitution of some cations (e.g. replacement of $\mathrm{Ba}$ by $\mathrm{Sm}$ in $\mathrm{Sm}_{1+y} \mathrm{Ba}_{2-y} \mathrm{Cu}_{3} \mathrm{O}_{7-x}$ ). Three regions with different doping are usually distinguishable [3]; in overdoped and optimal doped regions the superconducting 
transition temperature, $T_{\mathrm{c}}$, changes slightly (plateau at $T_{\mathrm{c}} \approx 90 \mathrm{~K}$ ), however in underdoped region $T_{\mathrm{c}}$ falls quickly with decreasing doping. For $\mathrm{YBa}_{2} \mathrm{Cu}_{3} \mathrm{O}_{7-x}$ the overdoped region lies above oxygen concentration of $\approx 6.90$, the optimal doped region lies around 6.85 and below concentration $\approx 6.8$ the underdoped region lies. Of course, the location of these regions is slightly different for compounds with other $\mathrm{RE}$, however $T_{\mathrm{c}}$-plateau range contains optimally doped and overdoped regions, too. Transports properties of $\mathrm{REBa}_{2} \mathrm{Cu}_{3} \mathrm{O}_{7-x}$ compounds are significantly different in these three regions, e.g., thermoelectric power is negative in overdoped region, but at lower oxygen concentration it changes sign into positive [4-6].

In this paper, we present temperature dependence of the resistivity and some parameters which characterize normal and superconducting states for $\mathrm{REBa}_{2} \mathrm{Cu}_{3} \mathrm{O}_{7-x}$ ceramic samples with different doping of $\mathrm{CuO}_{2}$ plane. All our samples have the superconducting transition temperature $T_{\mathrm{c}} \approx 90 \mathrm{~K}$ or slightly lower. The elements: $Y, E r, E u, G d, H o, D y$ and solid solutions $Y_{0.75} E_{0.25}, Y_{0.5} E_{0.5}$ have been used as $\mathrm{RE}$, and samples with substitution $\mathrm{Sm}_{1+y} \mathrm{Ba}_{2-y} \mathrm{Cu}_{3} \mathrm{O}_{7-x}$ [7] have also been investigated. The ceramic samples are textured (as X-ray measurements show), $a b$ plane is mainly in pellet surface and therefore temperature dependence of the resistivity $\rho(T)$ is close to that of single crystals in $a b$ plane, as it is often observed in the cuprates [8]. However, the $\rho$ values of ceramics are higher because of carriers scattering on grain boundaries. Temperature dependence of the normal state resistivity of these high- $T_{\mathrm{c}}$ superconductors changes its character with doping of $\mathrm{CuO}_{2}$ plane. At low temperatures distinct deviation from the linear dependence of the resistivity is always observed. The $\rho(T)$ dependence is exactly linear only for optimally doped samples above $200 \mathrm{~K}$. The Bloch-Grüneisen formula, which represents the temperature dependence of resistivity based on the electron-phonon scattering, is not sufficient to describe these experimental courses. Lately, the most frequently discussed mechanism is scattering due to spin fluctuation in the $\mathrm{CuO}_{2}$ plane (referred to as "spin gap") $[3,9,10]$.

\section{Experimental}

$\mathrm{REBa}_{2} \mathrm{Cu}_{3} \mathrm{O}_{7-x}$ compounds were prepared by the standard solid state reaction procedure from high purity $\mathrm{RE}_{2} \mathrm{O}_{3}, \mathrm{BaCO}_{3}$ and $\mathrm{CuO}$ powders. A well mixed powder was calcinated in air at $950^{\circ} \mathrm{C}$ for $12 \mathrm{~h}$. Next, the material was grinded, cold-pressed into a pellet and sintered again at $950^{\circ} \mathrm{C}$ in flowing oxygen. Only samples of $\mathrm{Sm}_{1+y} \mathrm{Ba}_{2-y} \mathrm{Cu}_{3} \mathrm{O}_{7-x}$, where the $\mathrm{Sm}$ ions partially substitute $\mathrm{Ba}$, were prepared by the process of citrate pyrolysis [7]. Finally, all the pellets were heated at $400-460^{\circ} \mathrm{C}$ for $20 \mathrm{~h}$ in flowing oxygen and then cooled to room temperature. The oxygen concentration in the samples was determined by the thermogravimetric or iodometric method with the accuracy of \pm 0.05 and it was in the range 6.7-7.0. The $\mathrm{X}$-ray powder analysis of the samples proved them to be of single-phase orthorhombic structure. The resistivity was measured by the conventional dc four-contact method on bar-shaped samples with dimensions of about $1 \times 2 \times 10 \mathrm{~mm}^{3}$, cut out from pellets parallel to their surface and with $\mathrm{Cu}$ wires attached to the samples by silver paste. The superconducting transition temperature, $T_{\mathrm{c}}$, was obtained from resistive transition curve as a temperature of 0.5 normal state resistivity value. The 
resistivity of the samples was measured in a range from $T_{\mathrm{c}}$ to $300 \mathrm{~K}$. The thermopower, $S$, was measured by the method described in Ref. [11]. Magnetization measurements in low magnetic fields (at $T=4.2 \mathrm{~K}$ ) allowed to determine the lower critical field, $H_{\mathrm{cl}}(0)$ as a field at which deviation from linearity of magnetization begins [12] (the $H_{\mathrm{cl}}\left(T=4.2 \mathrm{~K}\right.$ ) is very close to $H_{\mathrm{cl}}(0)$ ). In ceramic samples two such characteristic points appear. First point can be related to the lower critical field in the intergrain area or weakly coupled Josephson junctions, $H_{\mathrm{c} 1}^{\mathrm{J}}$ [13]. The other one, well above the former, is the intragrain lower critical field, $H_{\mathrm{c} 1}$.

\section{Results and discussion}

Twelve different samples were investigated and the six most characteristic ones were chosen for presentation. The basic data for the chosen samples are displayed in Table. Samples have been classified as belonging to different regions of doping of the $\mathrm{CuO}_{2}$ plane (underdoped, optimally doped or overdoped) according to values of $T_{\mathrm{c}}$, oxygen concentration, thermopower and Hall carrier concentration for some samples (e.g. $\mathrm{Sm}_{1+y} \mathrm{Ba}_{2-y} \mathrm{Cu}_{3} \mathrm{O}_{7-x}$ ). The values of the resistivity at $300 \mathrm{~K}, \rho_{300}$, for ceramic samples are not related to oxygen concentration because of important role of the scattering of carriers on the grain boundaries. However, the resistivity ratio $\rho_{300} / \rho_{100}$ and the derivative $(\mathrm{d} \ln \rho / \mathrm{d} T)_{300}(=(\mathrm{d} \rho / \mathrm{d} T) / \rho$ at $300 \mathrm{~K}$ ) values are clearly a function of the sample composition.

TABLE

Basic parameters characterizing the $\mathrm{REBa}_{2} \mathrm{Cu}_{3} \mathrm{O}_{7-x}$ samples. Superconducting transition temperature, $T_{c}$, width of the transition, $\Delta T_{c}$, values of $\rho_{300} / \rho_{100}$, and $(\mathrm{d} \ln \rho / \mathrm{d} T)_{300}$ have been obtained from resistivity measurements, $\rho(T)$. $S_{300}$ is the value of the thermopower at $300 \mathrm{~K} . H_{\mathrm{c} 1}^{\mathrm{J}}(0)$ and $H_{\mathrm{c} 1}(0)$ are intergrain and intragrain lower critical field, respectively, extrapolated to $T=0 \mathrm{~K}$.

\begin{tabular}{c|c|c|c|c|c|c|c|c|c}
\hline \hline No. & $\mathrm{X}$ & $\begin{array}{c}T_{c} \\
{[\mathrm{~K}]}\end{array}$ & $\begin{array}{c}\Delta T_{\mathrm{c}} \\
{[\mathrm{K}]}\end{array}$ & $\begin{array}{c}\rho_{300} \\
{[\mathrm{~m} \Omega \mathrm{cm}]}\end{array}$ & $\rho_{300} / \rho_{100}$ & $\begin{array}{c}(\mathrm{d} \ln \rho / \mathrm{d} T)_{300} \\
\times 10^{3}\left[\mathrm{~K}^{-1}\right]\end{array}$ & $\begin{array}{c}S_{300} \\
{[\mu \mathrm{V} / \mathrm{K}]}\end{array}$ & $\begin{array}{c}H_{c 1}^{\mathrm{J}}(0) \\
{[\mathrm{Oe}]}\end{array}$ & $\begin{array}{c}H_{c 1}(0) \\
{[\mathrm{Oe}]}\end{array}$ \\
\hline 1 & $\mathrm{~A}$ & 92.2 & 0.6 & 1.1 & 2.5 & 3.1 & -1.1 & 34 & 510 \\
& $\mathrm{~B}$ & 91.4 & 1.5 & 0.7 & 2.5 & 3.0 & +0.3 & & \\
\hline 2 & $\mathrm{C}$ & 91.6 & 1.1 & 1.4 & 2.1 & 2.3 & +4.7 & & \\
& $\mathrm{D}$ & 93.7 & 1.4 & 1.3 & 2.1 & 2.3 & +7.0 & 18 & 304 \\
\hline 3 & $\mathrm{E}$ & 87.8 & 6 & 1.7 & 1.9 & 1.8 & +23 & & \\
& $\mathrm{~F}$ & 82.7 & 6 & 1.9 & 1.8 & 1.6 & +15 & 6 & 185 \\
\hline
\end{tabular}

$\mathrm{X}=$ Composition, $\mathrm{A}=\mathrm{ErBa}_{2} \mathrm{Cu}_{3} \mathrm{O}_{6.95}, \mathrm{~B}=\mathrm{YBa}_{2} \mathrm{Cu}_{3} \mathrm{O}_{6.92}, \mathrm{C}=\mathrm{Y}_{0.75} \mathrm{Er}_{0.25} \mathrm{Ba}_{2} \mathrm{Cu}_{3} \mathrm{O}_{6.86}$,

$\mathrm{D}=\mathrm{EuBa}_{2} \mathrm{Cu}_{3} \mathrm{O}_{6.83}, \mathrm{E}=\mathrm{EuBa}_{2} \mathrm{Cu}_{3} \mathrm{O}_{6.75}, \mathrm{~F}=\mathrm{Sm}_{1.15} \mathrm{Ba}_{1.85} \mathrm{Cu}_{3} \mathrm{O}_{7.0}$.

The group No. 1 in Table consists of two samples with oxygen concentration above 6.9 and with low values of the thermopower at $300 \mathrm{~K}, S_{300}$ (comparison with the dependence of $S$ on $x$ presented in [4] would indicate a little higher concentration), i.e., the samples are in the overdoped region [6]. The values of the $T_{c}$ for the next two samples (group No. 2) are still close to $90 \mathrm{~K}$ but they show lower oxygen concentration, considerably higher $S_{300}$ values, lower values of the resistivity ratio $\rho_{300} / \rho_{100}$ and the derivative $(\mathrm{d} \ln \rho / \mathrm{d} T)_{300}$ than those from 
solid solution $\mathrm{Y}_{0.75} \mathrm{Er}_{0.25}$ as $\mathrm{RE}$ element does not clearly differentiate the sample. The last two samples in Table (group No. 3) exhibit $T_{c}$ below the plateau value, therefore they are in underdoped region. The $\mathrm{EuBa}_{2} \mathrm{Cu}_{3} \mathrm{O}_{6.75}$ sample lies in this region due to low oxygen concentration, whereas the $\mathrm{Sm}_{1.15} \mathrm{Ba}_{1.85} \mathrm{Cu}_{3} \mathrm{O}_{7.0}$ sample is underdoped due to partial substitution of $\mathrm{Ba}^{2+}$ by $\mathrm{Sm}^{3+}$ cations. Comparison of $S_{300}$ values for these three groups (Table) indicates also that the last group of samples (No. 3) lies in the underdoped region. This means that both oxygen nonstoichiometry and cation substitution result in a similar change of the doping level of $\mathrm{CuO}_{2}$ plane.

Temperature dependences of the resistivity, $\rho(T)$, of all samples exhibit some deviations from linear courses and they are analysed below. The peculiarities of $\rho(T)$ dependences are better visible on plots of $\mathrm{d} \rho / \mathrm{d} T$ versus $T$. Figure 1 shows the $T$-dependence of the derivative, $\mathrm{d} \rho / \mathrm{d} T$, for samples which are described in Table. Values of the derivative are calculated from experimental points distanced

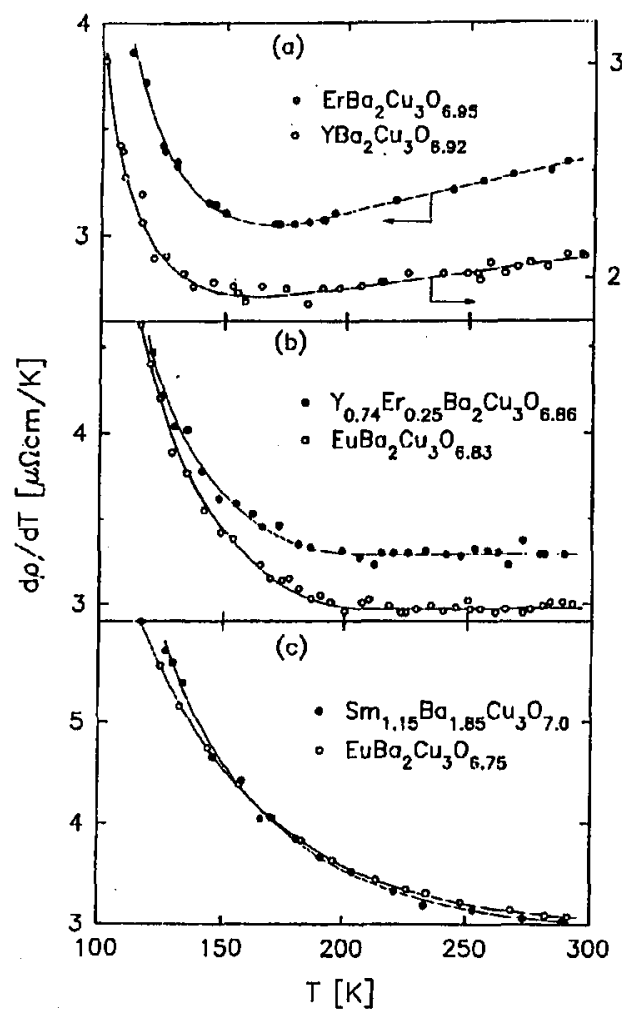

Fig. 1. The temperature dependence of the derivative, $d \rho / d T$, for $\mathrm{REBa}_{2} \mathrm{Cu}_{3} \mathrm{O}_{7-x}$ ceramics, with variously doped $\mathrm{CuO}_{2}$ plane: (a) overdoped, (b) optimally doped, (c) underdoped. The continuous lines are guides for eye only. 
by $\Delta T \approx 2 \div 4 \mathrm{~K}$. The shapes of $\mathrm{d} \rho / \mathrm{d} T$ above $200 \mathrm{~K}$ differ for these three groups. For overdoped samples (Fig. 1a) the derivatives, $\mathrm{d} \rho / \mathrm{d} T$, increase linearly with the increasing temperature $\left(\mathrm{d}^{2} \rho / \mathrm{d} T^{2}>0\right)$ in the range of $190-300 \mathrm{~K}$, therefore a component of the resistivity proportional to $T^{2}$ appears here. The presence of the component $\rho \sim T^{2}$ is predicted in overdoped region by the mean field phase diagram of $\mathrm{CuO}_{2}$ plane $[3,9]$ (i.e., a Fermi-liquid behaviour becomes important). Both samples from group No. 2 (Fig. 1b), which are in optimally doped region show exactly constant values of the $\mathrm{d} \rho / \mathrm{d} T$ in the range of $210-300 \mathrm{~K}\left(\mathrm{~d}^{2} \rho / \mathrm{d} T^{2}=0\right)$. Hence, $\rho(T)$ could be described by a simple relation $\rho(T)=\rho_{0}+a T$, in agreement with the phase diagram $[3,11]$, which predicts a linear $T$-dependence of the resistivity in optimally doped region and defines the material as strange metal. The derivatives, $\mathrm{d} \rho / \mathrm{d} T$, for samples from group No. 3 (Fig. 1c), which are in underdoped region, decrease with increasing temperature $\left(\mathrm{d}^{2} \rho / \mathrm{d} T^{2}<0\right)$. Therefore, the deviation from linearity of the $\rho(T)$ is opposite to that for overdoped samples. In the underdoped region the phase diagram $[3,9,10]$ predicts electron scattering mechanism due to spin fluctuations.

Below $200 \mathrm{~K}$ the derivative, $\mathrm{d} \rho / \mathrm{d} T$, changes similarly for all samples (Fig. 1), strongly increasing with decreasing temperature $\left(\mathrm{d}^{2} \rho / \mathrm{d} T^{2}<0\right)$, i.e., the decrease in resistivity is considerably faster than linear.

Temperature dependence of the resistivity for simple metals is essentially different than for $\mathrm{REBa}_{2} \mathrm{Cu}_{3} \mathrm{O}_{7-x}$ compounds. Namely, the derivative $\mathrm{d} \rho / \mathrm{d} T$ for metals is almost constant in a wide temperature range, whereas for these compounds the derivative changes in different way, depending on the doping level of the $\mathrm{CuO}_{2}$ plane. At low temperatures (considerably below the Debye temperature) the derivative for metals decreases very fast with the decreasing temperature $\left(\mathrm{d}^{2} \rho / \mathrm{d} T^{2}>0\right)$, whereas for the compounds the $\mathrm{d} \rho / \mathrm{d} T$ value increases $\left(\mathrm{d}^{2} \rho / \mathrm{d} T^{2}<0\right)$. Therefore, the Bloch-Grüneisen formula describing the dependence $\rho(T)$ for metals is not appropriate for the high- $T_{\mathrm{c}}$ superconductors.

Change of the doping of $\mathrm{CuO}_{2}$ plane strongly influences the values of lower critical field $H_{\mathrm{c} 1}$ diminishing it several times when the doping decreases (see Table) and this effect shows that some degradation of superconductivity appears in the compounds when the hole concentration is decreased. Thus, large differences in the values of the lower critical field $H_{\mathrm{c} 1}$ for $90 \mathrm{~K} \mathrm{YBa}_{2} \mathrm{Cu}_{3} \mathrm{O}_{7-x}$-type samples presented in literature [14] might be caused by small differences in oxygen concentration or other defects which influence the doping of $\mathrm{CuO}_{2}$ plane.

\section{Conclusion}

The temperature dependence of the normal state resistivity, $\rho(T)$, of $\mathrm{REBa}_{2} \mathrm{Cu}_{3} \mathrm{O}_{7-x}$ compounds exhibits clear deviation from linearity. We have revealed that at temperatures above $200 \mathrm{~K}$ this deviation apparently depends on doping of the $\mathrm{CuO}_{2}$ plane. The derivative, $\mathrm{d} \rho / \mathrm{d} T$, for overdoped samples increases linearly with the increasing temperature, whereas it decreases when the temperature increases for underdoped samples. The optimally doped samples show exactly linear $\rho(T)$ dependence. At low temperatures, below $200 \mathrm{~K}$, the deviation from the linear $\rho(T)$ dependence is similar for all samples. The derivative $\mathrm{d} \rho / \mathrm{d} T$ increases very fast when temperature decreases. 
These dependences of the $\rho(T)$ differ considerably from the temperature dependence of the resistivity of simple metals, therefore the classical electron-phonon scattering model is not sufficient for high- $T_{\mathrm{c}}$ superconductors. Some features of the $\rho(T)$ dependence of the $\mathrm{REBa}_{2} \mathrm{Cu}_{3} \mathrm{O}_{7-x}$ compounds are in qualitative agreement with predictions of mean field phase diagram for the $\mathrm{CuO}_{2}$ plane [3, 9], namely, the linear dependence of $\rho(T)$ in optimally doped region and the appearance of the resistivity component proportional to $T^{2}$ in the overdoped region.

The lower critical field $H_{\mathrm{c} 1}$ shows strong dependence on the hole concentration, i.e., value of $H_{\mathrm{c} 1}$ strongly decreases when the doping level of the $\mathrm{CuO}_{2}$ plane decreases.

\section{Acknowledgments}

We would like to thank Z. Damm and Z. Bukowski for preparation of some of the samples, and T. Plackowski and J. Olejniczak for valuable discussions.

\section{References}

[1] Y. Iye, in: Physical Properties of High Temperature Superconductors, Ed. D.M. Ginsberg, World Scientific, Singapore 1992, p. 285.

[2] J.D. Jorgensen, D.G. Hinks, P.G. Radaelli, Shiyou Pei, P. Lightfoot, B. Dabrowski, C.U. Segre, B.A. Hunter, Physica C 185-189, 184 (1991).

[3] B. Bucher, P. Steiner, J. Karpinski, E. Kaldis, P. Wachter, Phys. Rev. Lett. 70, 2012 (1993).

[4] P.J. Ouseph, M. Ray O'Bryan, Phys. Rev. B 41, 4123 (1990).

[5] Z. Henkie, P.J. Markowski, R. Horyn, Z. Bukowski, J. Klamut, Phys. Status Solidi $B$ 146, K131 (1988).

[6] K. Matsuura, T. Wada, Y. Yaegasi, S. Tajima, H. Yamauchi, Phys. Rev. B 46, 11923 (1992).

[7] Z. Bukowski, K. Rogacki, R. Horyń, in: Proc. 4rd Int. Conf. ICMAS'92, Paris 1992, Ed. A. Niku-Lari, IITT-International, Gourney sur Marne 1992, p. 181.

[8] H. Takagi, B. Batlogg, H.L. Kao, R.J. Cava, J.J. Krajewski, W.F. Peck, Jr., Phys. Rev. Lett. 69, 2975 (1992).

[9] N. Nagaosa, P.A. Lee, Phys. Rev. B 45, 966 (1992).

[10] T. Ito, K. Takenaka, S. Uchida, Phys. Rev. Lett. 70, 3995 (1993).

[11] C. Sułkowski, K. Rogacki, Z. Bukowski, R. Horyń, E. Trojnar, Physica C 153-155, 1337 (1988).

[12] C. Sułkowski, K. Rogacki, Phys. Status Solidi A 125, K101 (1991); K. Rogacki, C. Sułkowski, Mod. Phys. Lett. 6, 41 (1992).

[13] J.R. Clem, in Proc. $M^{2} H T S C$, Interlaken (Switzerland) 1988, Eds. J. Müller, J.L. Olsen, North-Holland, Amsterdam 1988, p. 50.

[14] A. Umezawa, G.W. Crabtree, K.G. Vandervoort, U. Welp, W.K. Kwok, J.Z. Liu, Physica C 162-164, 733 (1989); Dond-Ho Wu, S. Sridhar, Phys. Rev. Lett. 65, 2074 (1990). 